\title{
Les projets populaires
}

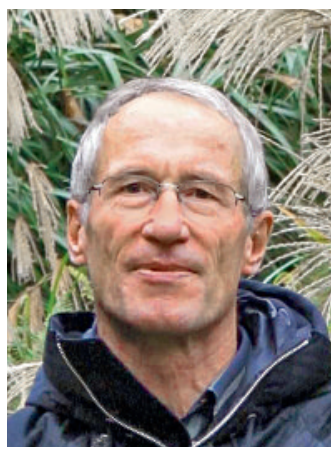

Erhard Taverna
Le nombre de mouvements citoyens ou populaires est en augmentation depuis des années. Ils sont généralement lancés par des individus assez jeunes, à qui Internet fournit une plateforme bon marché et difficile à contrôler sur laquelle exposer leurs préoccupations. Lorsqu'il s'agit d'attaquer les dictatures, leur action est accueillie de manière favorable, voire soutenue. On oublie parfois que plus près de nous aussi, de plus en plus d'initiatives citoyennes et de mouvements sociaux s'organisent en dehors de toute appartenance à des partis politiques. Ces mouvements concernent souvent des questions écologiques. Quarante ans après sa parution, le rapport du Club de Rome est plus que jamais d'actualité. Alors que les problèmes tels que la course aux armements, la pollution environnementale, l'explosion démographique et la stagnation économique évoqués à l'époque par U Thant alors qu'il était secrétaire général des Nations Unies se sont aggravés, des menaces supplémentaires sont apparues, par exemple le changement climatique. Nombreux sont ceux qui doutent de la capacité des institutions démocratiques à répondre de manière efficace à ces enjeux. Nous prenons conscience du fait que nous avons atteint les limites de la croissance et que des changements structurels profonds de notre mode de niveau sont inéluctables. La politique guidée par la volonté de la majorité n'encourage que rarement la gestion durable pourtant souvent citée; dans les pays riches, les coûts écologiques et sociaux sont bien plus souvent rejetés vers les états plus faibles. Par le passé, la croissance à venir permettait de surmonter toutes les difficultés: une stratégie alliant pillage et destruction. La croyance dans le progrès s'effrite quand le sens de l'existence n'a plus la priorité sur l'augmentation du niveau de vie et que de nouvelles questions existentielles se posent. Churchill décrivait à une époque la démocratie comme «un mauvais système, mais le moins mauvais de tous les systèmes». La démocratie n'est pas une institution réservée uniquement aux périodes fastes, et exploitée par les candidats aux élections et les tacticiens opportunistes. Elle doit résister en temps de crise, et beaucoup ne l'en croient justement plus capable. Les mouvements populaires proposent-ils une issue? Les «citoyens en colère» sont-ils la solution? Optimistes, certains comptent sur l'influence de réseaux informatiques mondiaux regroupant des internautes engagés, tandis que d'autres ne voient dans ces actions que les illusions d'une société dépassée qui préserve ses acquis. Il est toujours intéressant de découvrir les divers points de vue sur Internet.

Le mouvement «Zeitgeist» est un phénomène de masse qui regroupe des millions de personnes et qui, depuis sa création à l'automne 2008, affiche comme objectif une «économie basée sur les ressources», avec une approche systémique qui propose une réponse à toutes les questions actuelles. Fondé par le réalisateur américain Peter Joseph, il a donné naissance à une organisation mondiale non centralisée qui voit ses membres se regrouper en «chapitres» par le biais des forums, de chats vocaux, du podcasting, de Facebook, de Skype et des e-mails. Des individus se réunissent ainsi au niveau régional pour organiser des réunions, des actions et des initiatives. D'éminents experts travaillant dans tous les domaines scientifiques diffusent sur Youtube, au moyen de courts métrages, leurs messages et opinions sur des thèmes tels que l'énergie, les transports, la production des denrées alimentaires, la santé, la construction urbaine, l'économie monétaire, etc. Le maître lui-même, qui garde secret son nom de famille, donne des interviews et des cours sur le travail équitable, les nouvelles formes de vie et la suppression de la société monétaire. Un porte-parole très âgé formule l'idée d'une manière très succincte: «This shit got's to go» (Cette merde doit disparaître). Les adhérents du mouvement sont convaincus que le système monétaire basé sur le profit et guidé par la croissance est le principal responsable de la destruction du monde naturel. Des solutions de remplacement reposant sur les technologies déjà existantes sont proposées pour chaque critique exprimée envers un élément de la situation actuelle. Les cours méritent d'être écoutés. Ce sont des prises de position directes, percutantes et engagées, quelquefois aussi, bien que plus rarement, naïves ou obscures. Les mises en œuvre prévues sont généralement optimistes, rationnelles, témoignent de la foi dans les technologies et prennent critiques envers la religion. L'utopie anticapitaliste d'un monde juste n'est pas nouvelle en soi. Toutes les utopies partent du principe que la situation n'est pas telle qu'elle pourrait être. Les films sont passionnants et pleins d'idées, allant jusqu'à présenter des modèles de villes du futur autosuffisantes. Il n'est pas précisé qui est censé mettre en œuvre ces changements. Les installations en forme de cercle et leurs rues en éventail ressemblent aux villes résidentielles de l'absolutisme. Ce n'est plus un château qui se trouve au centre, mais un calculateur général qui répertorie et contrôle tout, du train à lévitation magnétique jusqu'aux tours pour la culture hydraulique situées en périphérie. Pour nous, destructeurs de l'environnement, ce sont des incitations à réfléchir ou à tout repenser fascinantes, un miroir de notre impuissance.

Les projets populaires sont tenaces. Nous ne changerons cependant pas aussi rapidement de notre plein gré. Pour les générations futures qui survivront, l'envie sera vraisemblablement le moindre des soucis. 